\title{
The 3D Numerical Study on Flow and Sediment Properties of a River with Grouped Spur Dikes
}

\author{
Xun $\operatorname{Han}^{1,2}$, Pengzhi Lin ${ }^{1, *}$, and Gary Parker $^{2}$ \\ ${ }^{1}$ SKLH, Sichuan University, China, 610065 \\ ${ }^{2}$ CEE, University of Illinois Urbana and Champaign, USA, 61801
}

\begin{abstract}
A 3D numerical model named NEWTANK is employed to investigate the flow motion and sediment transport in grouped spur dikes system. This model is based on the Navier-Stokes equations, adopting the Volume of Fluid (VOF) method to track the free surface motion, while the solid is described by using the Porous Media Method (PMM). The Large Eddy Simulation (LES) is applied to capture turbulence. In sediment calculation parts, the suspended load and bedload are treated separately but combined together to update bed variation eventually. The finite difference form and Two-step Projection Method are employed in the process of discretizing the governing equation. Several carefully selected flume experiments are introduced to verify this model's reliability before its application on the simulation of grouped spur dike case, and detailed flow characteristics and sediment properties are analyzed afterwards.
\end{abstract}

\section{Introduction}

The spur dikes, which are also known as groynes are kinds of very common hydraulic structures in river engineering, especially on some big rivers such as Mississippi River, Rhine River and Yellow River. Since 1960s, the flow motion and sediment transport around the spur dikes have become a hot research issue which attracts many scholars and engineers to contribute their passion and energy. In this problem, Flow separation and circumfluence behind the spur dikes result in strongly 3D turbulence, while the pressure distribution is non-hydrostatic, which are directly related to the sediment transport and local scour around the spur dike, and the eventual balance are reached as flow and sediment strongly coupled with each other. Therefore, most researchers especially in early stage usually conduct their research on isolated spur dike case with experimental methods (Rajaratnam and Nwachukwu[1]; Kuhnle and Alonso[2,3]; Duan et al. [4]; Kang and Yeo et al. [5]; Zhang et al. [6,7]; Uijttewaal[8]).

Benefit from the generation of information technology and numerical techniques, numerical simulation has gradually become an important way in this research area (Tingsanchali and Matheswaran[9]; Muneta and Shimizu[10]; Mayerle et al. [11]; Molls and Chaudhry et al. [12]; Ouillon and Dartus[13]; Nagata and Hosoda et al. [14]; Koken and Constantinescu[15,16]; Khosronejad et al. [17,18]). However, the research purposes of all

\footnotetext{
*Corresponding author: cvelinpz@scu.edu.cn
} 
these studies are still finding the mechanism of the fluid and sediment coupling in the process of scour under the condition of one single spur dike. Actually, it is more common that the spur dikes are constructed as the grouped spur dikes system in engineering application, and in the grouped spur dike systems, the motion of flow and sediment would be more complicated because of the interaction between the spur dikes. Therefore, these achievements on isolated spur dike case can provide fairly useful references, but studying the phenomenon of fluid motion and sediment transport in grouped spur dikes system is still needed.

Compared with the achievements of isolated spur dike case, the studies on grouped spur dike are much insufficient, and flume experiment and field measurement are the main methods (Bahar and Fukuoka[19]; Muto and Khaleduzzaman[20,21]; Tominaga and Ijima[22]; Engelhardt and Kruger[23]; Uijttewaal et al. [24]; Kurzke and Weitbrecht et al. [25]; Kang and Yeo et al. [26]), while the research systematicness is relatively poor. For the aspect of numerical simulation, Peng et al. [27,28] used a 3D numerical model with k- $\varepsilon$ turbulence module to study the flow structure in a double spur dikes system based on their work on isolated spur dike case before. McCoy, Constantinescu and Weber [29,30] employed the LES module into the research of pollutant dispersion in a double spur dikes system with a fixed bed and obtained fairly good agreement with experimental data. Karami and Basser et al. [31] compared their simulation with flume test on the flow properties and bed variations to verify their numerical tool.

To sum up, the available numerical model that can predict not only the flow motion but also the sediment transport is not abundant, resulting in unclear understanding on the mechanism of flow-sediment coupling in grouped spur dikes system. Therefore, here we illustrate an application of a fully 3D numerical model combined with the LES scheme and porous media method (PMM) on fluid motion of grouped spur dikes problems involving local scour and sediment moving. Firstly we introduce the model, and then present verification against laboratory experiments to verify this model's reliability, afterwards, this model is applied to investigate concerned hydrodynamic process and sediment transport phenomenon while some detailed analyses will be conducted accordingly.

\section{Numerical Method}

\subsection{Hydrodynamic Equations}

The motion of the incompressible fluid within and outside porous media can be described by the modified Navier-Stokes equations (Lin and Karunarathna[32]):

$$
\begin{gathered}
\frac{\partial \bar{u}_{i}}{\partial x_{i}}=0 \\
\frac{1}{n} \frac{\partial \bar{u}_{i}}{\partial t}+\frac{\bar{u}_{j}}{n^{2}} \frac{\partial \bar{u}_{i}}{\partial x_{j}}=-\frac{1}{\rho} \frac{\partial \bar{p}_{0}}{\partial x_{i}}+\frac{v}{n} \frac{\partial^{2} \bar{u}_{i}}{\partial x_{j} \partial x_{j}}-\bar{f}_{i}-\frac{1}{n^{2}} \frac{\partial \overline{u_{i}^{\prime \prime} u_{j}^{\prime \prime}}}{\partial x_{j}}
\end{gathered}
$$

Where $\bar{u}_{i}$ is the spatially averaged flow velocity in $i$ direction, $p_{0}$ is the effective pressure, $\rho$, $v$ and $n$ are density, kinematic viscosity of fluid and effective porosity, respectively. The force $\bar{f}_{i}$ is caused by the presence of porous material in flow, which includes inertia and drag forces $\bar{f}_{i}=\bar{f}_{I i}+\bar{f}_{D i}$ 
The concept of two-phase flow is introduced into this model, and the three dimensional PLIC-VOF (Gueyffier and Nadim et al. [33]) method which originates from Youngs' theory [34] is adopted to track the free surface motion. The VOF transport equation is:

$$
\frac{\partial F}{\partial t}+\frac{\partial\left(\bar{u}_{i} F\right)}{\partial x_{i}}=0
$$

Where $F$ is the volume of fluid function, with the help of which, the density of fluid can be rewritten as $\rho=F \rho_{w}+(1-F) \rho_{a}$, Where the subscript $w$ and $a$ mean water and air.

In present model, the Large Eddy Simulation (LES) is employed to capture turbulence transport and dissipation. After being filtered by the spatial filter Top-Hat function, the subgrid stress terms appear in the momentum equations, which can be modelled by the Smagorinsky sub-grid scale model (Smagorinsky[35]):

$$
\tau_{i j}-\frac{1}{3} \tau_{k k} \delta_{i j}=-2 \rho v_{t}\left(\frac{\partial \bar{u}_{i}}{\partial x_{j}}+\frac{\partial \bar{u}_{j}}{\partial x_{i}}\right)
$$

Where $v_{t}=C_{s} \Delta^{2}|\bar{S}|$ is the eddy viscosity. $C_{s}$ is the Smagorinsky coefficient. $\Delta=\sqrt[3]{\Delta x \Delta y \Delta z}$ is the filter size calculated by control volume $\Delta x, \Delta y$ and $\Delta z \cdot|\bar{S}|=\sqrt{2 \bar{S}_{i j} \bar{S}_{i j}}$ is the filtered strainrate tensor. The Smagorinsky coefficient $C s$ is usually a constant which takes the value between 0.1 and 0.2. However, for dealing with high Re number turbulent flows like those in this study, the dynamic Smagorinsky model (Germano and Piomelli et al. [36]) is a more appropriate option, which is embedded into the original Smagorinsky model letting the dynamic Smagorinsky coefficient $C_{D}$ evolves in time and space as a function of the flow field, and the optimal value is selected to minimize the mean square error between the resolved stress at the grid filter and the test filter.

\subsection{Sediment formula}

The suspended load concentration is calculated by the advection-diffusion equation, from which the volumetric concentration $c$ can be calculated across the whole computational domain:

$$
\frac{\partial c}{\partial t}+u \frac{\partial c}{\partial x}+v \frac{\partial c}{\partial y}+\left(w-w_{s}\right) \frac{\partial c}{\partial z}=\frac{\partial}{\partial x}\left(\varepsilon_{s x} \frac{\partial c}{\partial x}\right)+\frac{\partial}{\partial y}\left(\varepsilon_{s y} \frac{\partial c}{\partial y}\right)+\frac{\partial}{\partial z}\left(\varepsilon_{s z} \frac{\partial c}{\partial z}\right)
$$

Where $u, v, w$ is the flow velocity in $x, y, z$ direction. $\varepsilon_{s x}, \varepsilon_{s y}$ and $\varepsilon_{s z}$ is the sediment mixing coefficient, which relates to the fluid mixing coefficient (van Rijn [37]). $w_{s}$ is the fall velocity of sediment in a suspension.

Abdel and van Rijn's relation [38] is introduced to describe the bed load transport rate:

$$
q_{b}= \begin{cases}0.053 \sqrt{(s-1) g d_{50}^{3}} \frac{T^{2.1}}{D_{*}^{0.3}} & T<3 \\ 0.100 \sqrt{(s-1) g d_{50}^{3}} \frac{T^{1.5}}{D_{*}^{0.3}} & T \geq 3\end{cases}
$$

Where $d_{50}$ is the median grain size of sediment, $T$ is the non-dimensional excess shear stress, $D *$ is the non-dimensional grain size, the expressions of which read:

$$
\begin{aligned}
D_{*} & =d_{50}\left[\frac{(s-1) g}{v^{2}}\right]^{\frac{1}{3}} \\
T & =\frac{\tau_{b}^{\prime}-\tau_{b, c r}}{\tau_{b, c r}}
\end{aligned}
$$


Where $\tau_{b}^{\prime}$ is the effective shear stress. $\tau_{b, c r}$ is the critical shear stress according to the fit of Shields diagram. To deal with the three dimensional cases of spur dikes problem, van Rijn's methods [39] to describe the effects of bed slope on critical shear stress and bed load transport rate are taken into account in this model.

The morphological variation is calculated based on the mass-balance equation:

$$
\left(1-\lambda_{p}\right) \frac{\partial \eta}{\partial t}=D_{a}-E_{a}-\frac{\partial q_{b x}}{\partial x}-\frac{\partial q_{b y}}{\partial y}
$$

Where $\lambda_{p}$ is the porosity of bed, $\eta$ is the bed elevation, $q_{b x}$ and $q_{b y}$ are the bed load transport rates in the $x$ and $y$ directions, $D_{a}$ and $E_{a}$ are the deposition and entrainment rates respectively.

\section{Model Verifications}

In this section, two sets of laboratory tests are employed to verify this numerical model's performances on different emphases.

\subsection{Isolated spur dike with fixed bed}

The flume experiment on isolated spur dike is carried out in Sichuan University (Fig.1), in which the flume is $4.0 \mathrm{~m} \times 0.6 \mathrm{~m} \times 0.368 \mathrm{~m}$ (length $\times$ width $\times$ height) with the slope of 0.0005 . The size of the spur dike is $12 \mathrm{~cm} \times 4 \mathrm{~cm} \times 1 \mathrm{~cm}$ (width $\times$ height $\times$ thickness). The water level is $7.6 \mathrm{~cm}$ while the inlet flow velocity is $12.6 \mathrm{~cm} / \mathrm{s}$. With the help of Particle Image Velocimetry (PIV) and PS tracer particle $(50 \mu \mathrm{m})$, Zhang measured the detailed 3D flow field around isolated spur dike and obtained much useful experiment data.
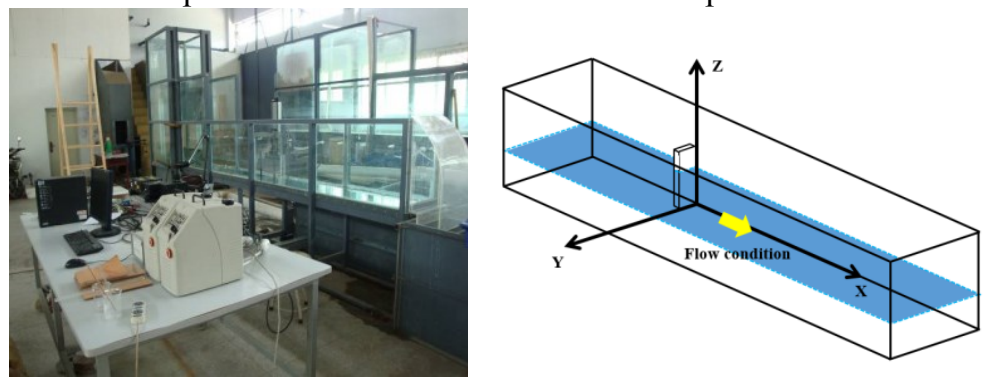

Fig.1. Photos of experiment and the coordinate

This numerical model is adopted to make corresponding simulation. The computational domains and flow conditions are same as the experimental tests, as well as the coordinate. After obtaining the numerical results, we compare them with experimental data taken by PIV. In this case, a vortex forms in the recirculation zone behind the spur dike, and the XY plane $\mathrm{Z}=0.05 \mathrm{~m}$ shows a good representation of the flow field, while compared with the transverse velocity component, the streamwise velocity component is dominant in this problem. Therefore, series of lines on this plane $(x=20 \mathrm{~mm}, x=40 \mathrm{~mm}, x=80 \mathrm{~mm}, x=160 \mathrm{~mm}$, $\mathrm{x}=240 \mathrm{~mm}, \mathrm{x}=480 \mathrm{~mm}$ ) are chosen, and then the comparison of time-average streamwise velocity component $U$ varying with y coordinate is conducted between numerical results and experimental data in Fig. 2. 


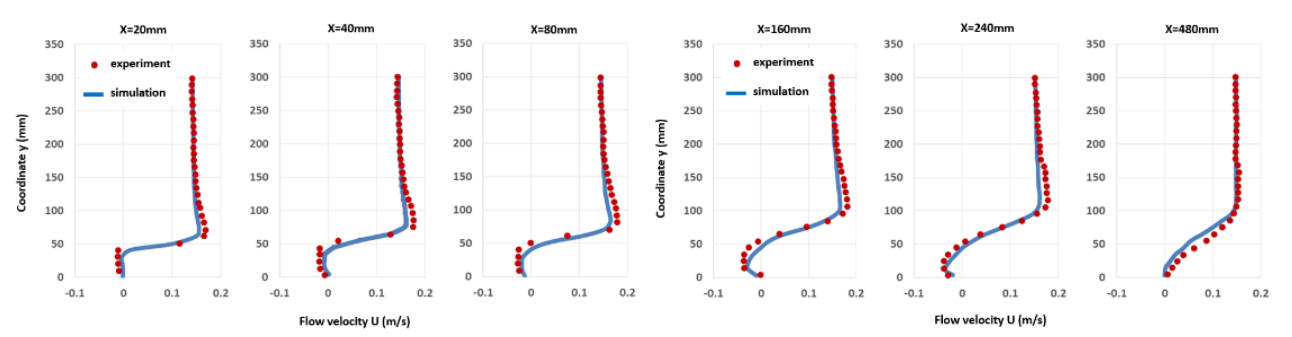

Fig.2. Comparison of time-average streamwise velocity component $U$ on XY plane in the nonsubmerged spur dike case

From the comparison, it can be found that the numerical results of the flow fields showing good agreements with the data measured by PIV in experimental tests, which proves the present numerical model can accurately capture the flow field and reproduce the vortexes formed around the spur dike.

\subsection{Evolution of the trench under open channel flow}

The sediment transport within grouped spur dikes system is actually a phenomenon of erosion and deposition, which is same as the mechanism of the trench evolution under open channel flow, therefore, to verify the sediment component's performance, an experimental test of a trench evolution under the water described in van Rijn's lecture [37] is introduced. Other verification cases of isolated spur dike could also be found in Xun's paper [40]. The flume is $30 \mathrm{~m} \times 0.5 \mathrm{~m} \times 0.7 \mathrm{~m}$ for the length, width and height respectively. The sediment particles on sand bed have median grain size $d_{50}=0.16 \mathrm{~mm}$. The water depth equals $0.39 \mathrm{~m}$, and the roughness height is $0.025 \mathrm{~m}$, while the flow velocity at the flume inlet is $0.51 \mathrm{~m} / \mathrm{s}$. The Schematic plot of this experiment is as shown in Fig.3. The sediment concentration along profile 1, 4, 6, 7 at the initial time and the bed elevation after 15 hours are measured in this experiment.

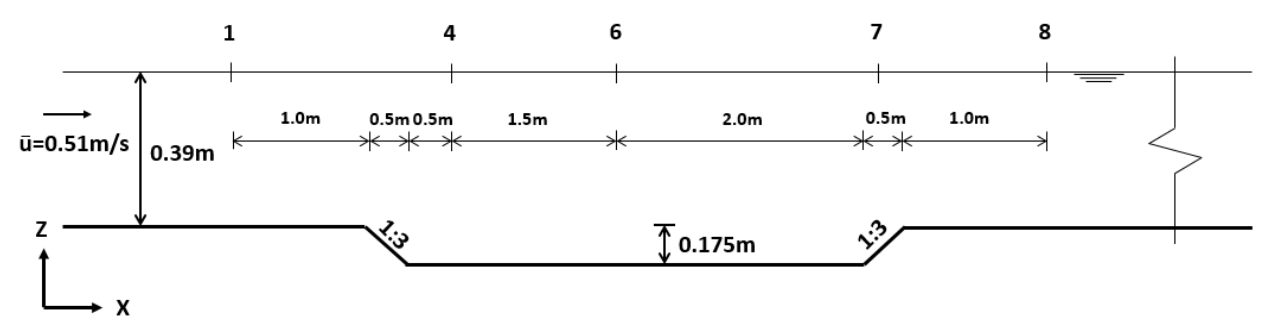

Fig.3. Schematic plot of the trench evolution experiment

The numerical simulation for this case is conducted totally based on the parameters in the flume experiment. The computational domain is set to $30 \mathrm{~m} \times 0.5 \mathrm{~m} \times 0.9 \mathrm{~m}$. The reference level a takes the value of $0.0125 \mathrm{~m}$. At the beginning, only the suspension component is switched on and the flow velocity and suspended load concentration is picked out, afterwards, the bed load and morphological variation component are switched to let the trench evolve and obtain the bed form information at 15 hours. Comparisons are then made accordingly (Fig.4, Fig.5). 

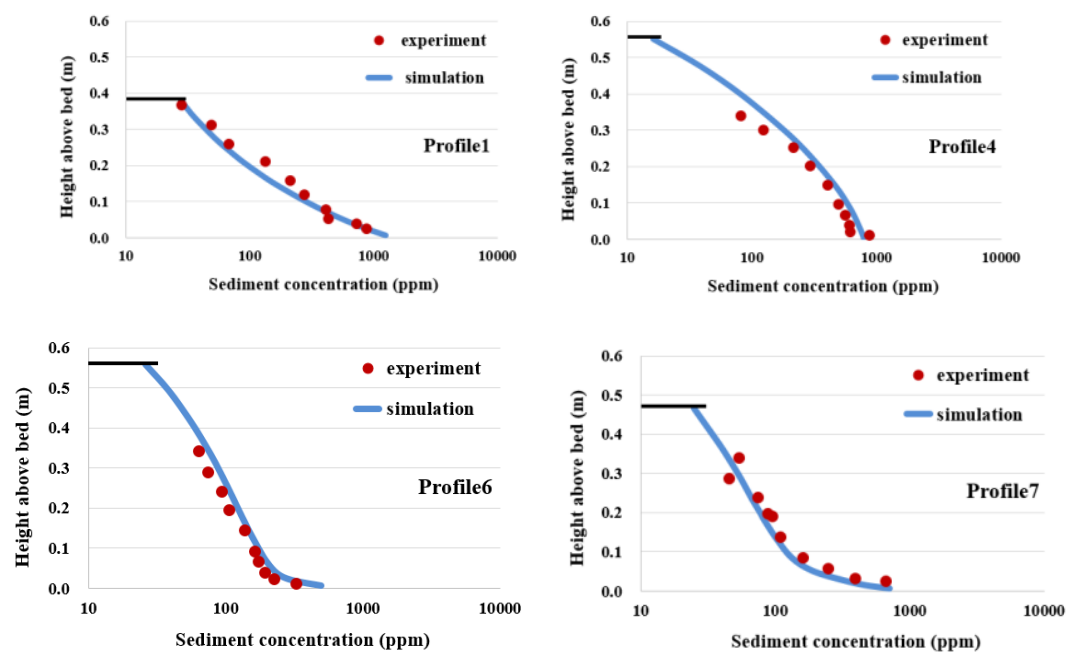

Fig.4. Comparison of the sediment concentration in the trench evolution case

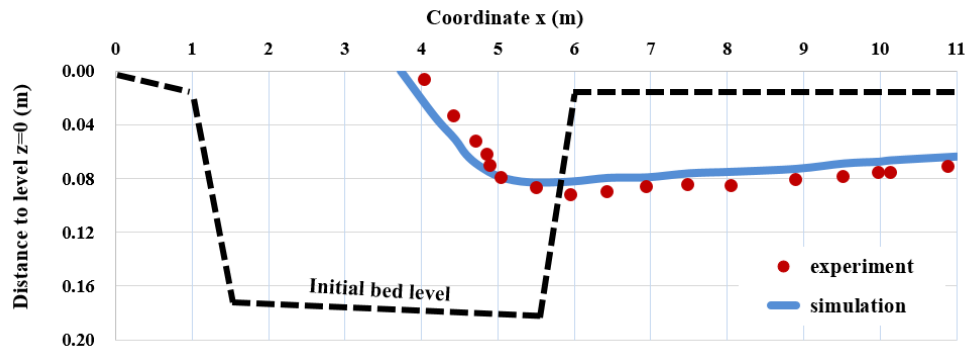

Fig.5. Comparison of bed elevation evolving for 15 hours in the trench evolution case

From the above comparisons, it can be concluded that the performance of this model's sediment component is sure, which means this model can reproduce the erosion and deposition under the flow condition, and good agreement can be obtained while comparing with the flume data.

\section{Grouped Spur Dikes Case}

In this section, the verified numerical model is applied to the study of flow properties and sediment moving in grouped spur dikes system with four non-submerged staggered spur dikes, which is the most common layout in river engineering. The numerical flume is $300 \mathrm{~m} \times 30 \mathrm{~m} \times 15 \mathrm{~m}$ for the length, width and height respectively with the bed slope 0.0001 . The sediment particles on sand bed have median grain size $d_{50}=0.7 \mathrm{~mm}$ and the thickness of the sand bed is $5 \mathrm{~m}$. The water depth equals $4.0 \mathrm{~m}$ while the flow discharge at the upstream is $188.77 \mathrm{~m} 3 / \mathrm{s}$ which is determined by the stage-discharge relation curve of uniform open channel flow without the spur dikes. Four spur dikes have the same size $6 \mathrm{~m} \times 7 \mathrm{~m} \times 1.8 \mathrm{~m}$ for the width, height and thickness respectively. All the other detailed information in the simulation can be found in Fig.6. 


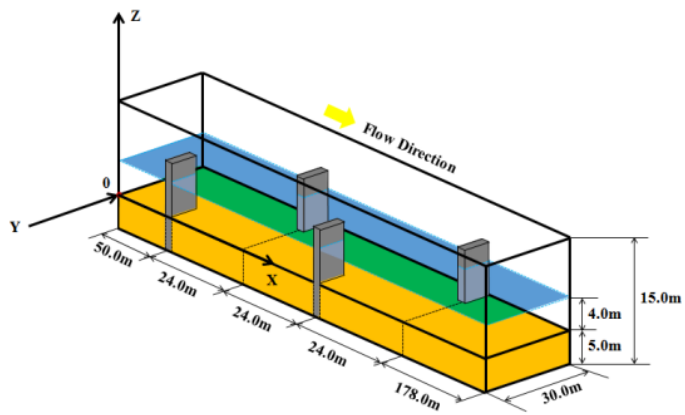

Fig.6. Schematic plot of the grouped spur dikes case

The non-uniform grid system is used and the numbers of the grids in three directions are 150, 40 and 70 respectively. The time step is adjusted automatically as the velocity changing in the simulation. At the beginning, only the hydrodynamic component is switched on to let it run until the flow field getting equilibrium, afterwards, the sediment component is switched on calculating the suspension as well as sand bed evolving to obtain the final bedform. At the equilibrium stage, the flow field on XY intersections $(\mathrm{z}=2.0 \mathrm{~m})$ are taken out as Fig.7.

$\begin{array}{llllllllllllll}\text { Velocity Magnitude (m/s): } & 0.0 & 0.3 & 0.5 & 0.8 & 1.0 & 1.3 & 1.5 & 1.8 & 2.0 & 2.3 & 2.5 & 2.8 & 3.0\end{array}$
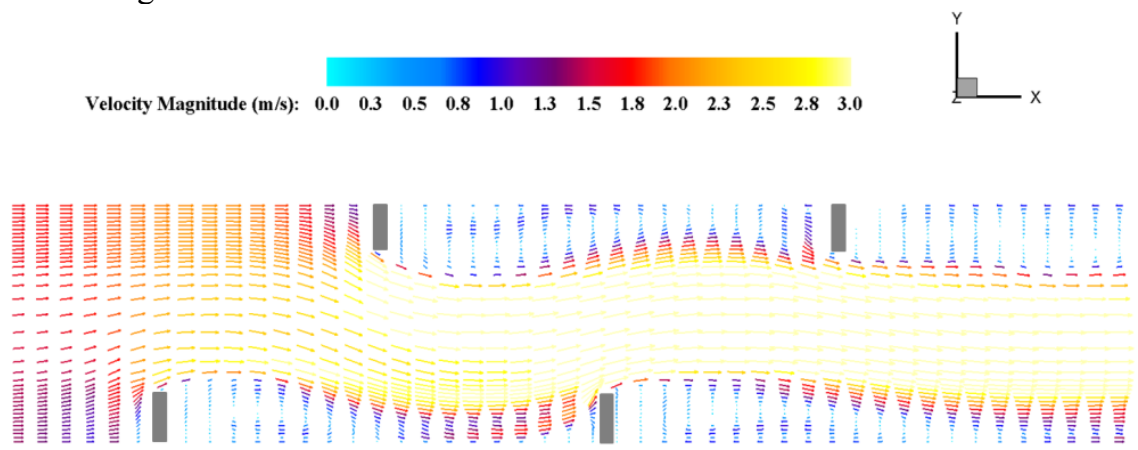

Fig.7. Flow field in the grouped spur dikes system (aerial view)

It can be seen that the main flow in the spur dikes system follow the path of S-shape, the velocity magnitude of which is accelerated from upstream to downstream, and the process of acceleration is relatively mild. For easy explanation, the four spur dikes are named with No.1-No.4 along the flow direction. Because of the S-shape main flow direction, the symmetrical axis of every vortex behind the spur dikes is not paralleled but having an angle with the sidewall which is not the same as the isolated spur dike case. In the vertical direction, we take the $\mathrm{XZ}$ plane of $\mathrm{y}=3.0 \mathrm{~m}$ as an example, which is near the right side wall and through the middle of No.1 and No.3 spur dikes. It can be seen (Fig.8, left) there is an intensive vortex in front of the No.1 spur dike digging the sand bed which is same as the isolated spur dike case, and due to the flow reflected by the No.2 spur dike, the flow intension at the downstream area between the No.1 and No.3 spur dikes is fairly strong, which also causes the bed erosion on the bed surface at that place. This phenomenon can be observed at the similar area between the No.2 and No.4 spur dikes. However, at the upstream area between double spur dikes, the flow intension is relatively weak due to the shelter effect of the spur dike in front of this zone. Moreover, except for the intensive vortex before No.1 spur dike, a small corner vortex also exists in front of every other spur dike. 

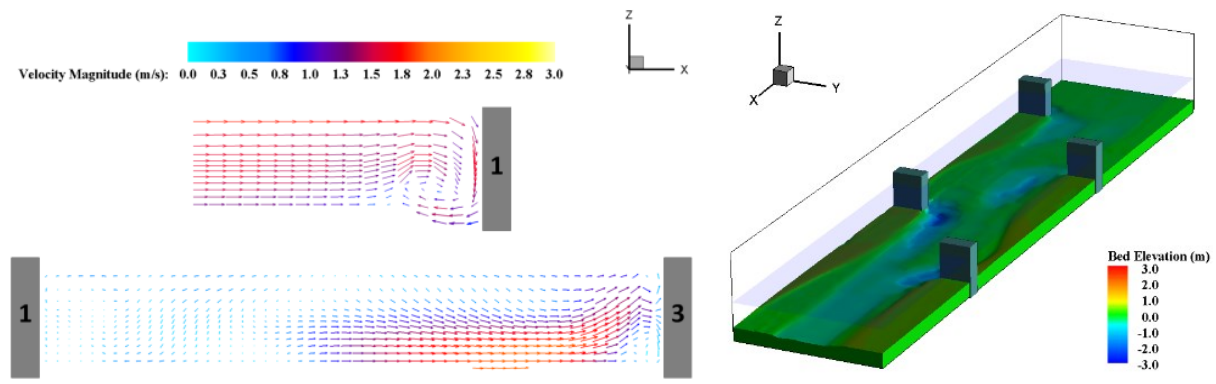

Fig.8. Flow field on plane $y=3.0 \mathrm{~m}$ and final bed elevation in the grouped spur dikes system

The right figure in Fig.8 shows the bed elevation at the equilibrium stage. It can be seen the bed erosion is also S-shape which is corresponding to the main flow path, while the erosion depth increases as the growing of flow velocity towards downstream, which is getting the maximum around the No.3 spur dike, and then decreases as the flow coming out of the grouped spur dike system. In front of the No.1 spur dike, the relatively intensive corner vortex causes erosion on the sand bed, which is different from the bedform in front of the No.2, No.3 and No.4 spur dikes where the corner vortexes are weak. From the figures of suspended load concentration at the intersections of $y=3 \mathrm{~m}$ and $\mathrm{y}=27 \mathrm{~m}$ (Fig.9), we can find the phenomenon that reflected flow by the No.2 and No.3 spur dikes entrain sediment away from the sand bed and cause the high concentration at the downstream area between double spur dikes, which is enriched in front of the next spur dike or comes into the main flow by the effect of turbulence. After the flow with high concentration sediment leaving the grouped spur dikes system, there is a deposition area at the downstream, which needs to be cared in engineering application.

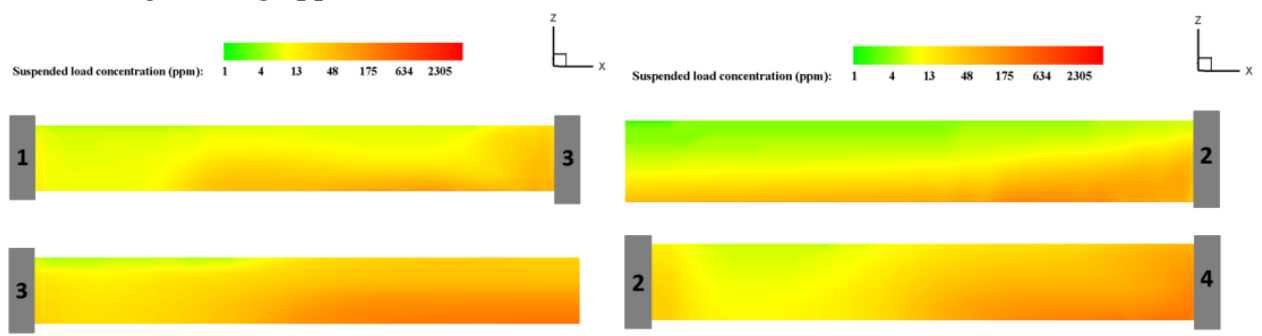

Fig.9. Suspended load concentration on the $X Z$ plane $(y=3 m$ and $y=27 m)$

\section{Conclusion}

In this research, the 3D numerical model which has the capability to predict the fluid motion and sediment transport simultaneously is introduced. After being verified on the performance of the hydrodynamic and sediment component by two sets of flume experiments respectively, this model is applied to study the properties of flow and sediment in the grouped spur dikes system. From the numerical results, it can be known that the flow structure shows fully $3 \mathrm{D}$ characteristics, which illustrates the main flow follows the $\mathrm{S}$ shape path while the large-scale vortexes behind the spur dikes form recirculation zone and the corner vortexes in front of the spur dikes also exist. The flow field causes the complicated distribution of suspended load and non-uniform erosion or deposition on sand bed. The numerical simulation demonstrates this model can be a powerful computational tool for scientific research and engineering design in the future. 


\section{References}

[1] Rajaratnam, N., Nwachukwu, B. A., J Hydraul Eng, 109(3), 463-480 (1983)

[2] Kuhnle, R. A., Alonso, C. V., Shields, F. D. Jr., J Hydraul Eng, 125(9), 972-978 (1999)

[3] Kuhnle, R., Jia, Y., Shields, F. D. Jr., J Hydraul Eng, 128(12), 1087-1093 (2002)

[4] Duan, J. G., J Hydraul Eng, 135(10), pp.803-811 (2009)

[5] Kang, J. G., Yeo, H. K., Kim, S. J., Ji, U., J Hydraul Res, 49(6), 728-735 (2011)

[6] Zhang, H., Nakagawa, H., Kawaike, K., Baba, Y. Int J Sediment Res, 24(1), 33-45(2009)

[7] Zhang, H., Nakagawa, H., Mizutani, H., Int J Sediment Res, 27(1), 141-157 (2012)

[8] Uijttewaal, W.S.J., J Hydraul Eng, 131(9), $782-791$ (2005)

[9] Tingsanchali, T., Maheswaran, S., J Hydraul Eng, 116(1), 71-86 (1990)

[10] Muneta, N., Shimizu, Y., Proceedings of JSCE, 497, 31-39 (1994)

[11] Mayerle, R., Toro, F. M., Wang, S. S. Y., J Hydraul Res, 33(2), 243-256 (1995)

[12] Molls, T., Chaudhry, M. H. Khan, K. W., Adv Water Resour, 118(4), 227-236 (1995)

[13] Ouillon, S., Dartus, D., J Hydraul Eng, 123(11), 962-970 (1997)

[14] Nagata, N., Hosoda, T., Nakato, T, J Hydraul Eng, 131(12), 1074-1087 (2005)

[15] Koken, M., J Hydraul Res, 49(6), 736-743 (2011)

[16] Koken, M., Constantinescu, G., Water Resour Res, 47, W12511 (2009)

[17] Khosronejad, A., Hill, C., Kang, S., Sotiropoulos, F., Adv Water Resour, 1(8), 191207 (2013)

[18] Khosronejad, A., Kozarek, J. L., Palmsten, M. L., Sotiropoulos, F., Adv Water Resour, 9(7), 309-332(2014)

[19] Bahar, S. M. H., Fukuoka, S., Ann J Hydraul Eng, JSCE, 43,671-676. (1999)

[20] Muto, Y., Kitamura, K., Khaleduzzaman, A.T.M., Nakagawa, H., Adv River Eng, 9 (2003)

[21] Muto, Y., Baba, Y., Fujita, I., River Flow 2002, 1213-1219 (2002)

[22] Tominaga, A., Ijima, K., Nakano, Y., 29th IAHR Congress (2001)

[23] Engelhardt, C., Kruger, A., Sukhodolov, A., Nicklisch, A., J of Plankton Res, 26, 1351-1366 (2004)

[24] Uijttewaal, W., Lehmann, D., van Mazijk, A., J Hydraul Eng, 127, 928-936 (2001)

[25] Kurzke, M., Weitbrecht, V., Jirka, G. H., River Flow 2002, 69-76 (2002)

[26] Kang, J. G., Yeo, H. K., Kim, S. J., Ji, U., Water Environ J, 25(2), 181-191(2011)

[27] Peng, J., Tamai, N., Kawahara, Y., J Sediment Res, 1(4). 25-29 (2002)

[28] Peng, J., Tamai, N., Kawahara, Y., Huang, G., 28th IAHR Congress (1999)

[29] McCoy, A., Constantinescu, S. G., Weber, L., Water Resour Res, 43, W05445 (2007)

[30] McCoy, A., Constantinescu, S.G., Weber, L., J Hydraul Eng, 134(2), 157-172 (2008)

[31] Karami, H., Basser, H., Ardeshir, A., Hosseini, H., Water Environ J, 28, 124-134(2012)

[32] Karunarathna, S. A. S. A., Lin, P., Coast Eng, 53, 845-855(2006)

[33] Youngs, D. L., Numerical methods for fluid dynamics, 24:273-85 (1982)

[34] Gueyffier, D., Li, J., Nadim, A., Scardovelli, R., Zaleski, S., J Comput Phys, 152:42356 (1999)

[35] Smagorinsky, J. S., Monthly Weather Review, 91, 99-164(1963)

[36] Germano, M., Piomelli, U., Moin, P., Cabot, W. H., Phys Fluid A, 3, 1760-1765(1991)

[37] van Rijn, L. C., Invited Lecture, Euromech Colloquium 192, West Germany (1985)

[38] Abdel-Fattah, S., Amin, A., van Rijn, L. C., J Hydraul Eng, 130(6), 488-500(2004)

[39] van Rijn, L. C., Aqua Publications (1993)

[40] Han, X., Lin, P. Z., Parker, G. J Hydraul Eng, (under review), (2018) 